\title{
Cenobamate for the Treatment of Focal Seizures
}

\author{
Pavel Klein, ${ }^{1}$ Louis Ferrari, ${ }^{2}$ and William E Rosenfeld ${ }^{3}$
}

1. Mid-Atlantic Epilepsy and Sleep Center, Bethesda, MD, USA; 2. SK Life Science, Inc., Paramus, NJ, USA;

3. Comprehensive Epilepsy Care Center for Children and Adults, St. Louis, MO, USA

E pilepsy is a common neurological disease. Anti-seizure medication (ASM) is the first-line treatment, beginning with monotherapy and, in uncontrolled epilepsy, progressing to combination therapy over time, with the goal of seizure freedom. Cenobamate is an oral ASM approved for the treatment of focal (partial-onset) seizures in adults. Two clinical studies (C013, C017) evaluated adjunctive cenobamate in uncontrolled focal epilepsy despite treatment with one to three ASMs. In C013, cenobamate $200 \mathrm{mg} /$ day, and in C017, cenobamate 100, 200, and $400 \mathrm{mg} /$ day reduced seizure frequency by $55.6 \%, 35.5 \%, 55.0 \%$, and 55.0\%, respectively. Up to 20\% more cenobamate-treated (C013: $200 \mathrm{mg} /$ day, C017: $400 \mathrm{mg} /$ day) patients achieved zero seizures versus placebo during the maintenance phase of therapy. Three cases of drug reaction with eosinophilia and systemic symptoms, including one fatality, were reported during clinical development with starting doses $\geq 50 \mathrm{mg} / \mathrm{day}$ and weekly up-titration. Safety data from C013, C017, and an open-label safety study (C021), demonstrated the most common adverse events associated with cenobamate were central nervous system-related. Given the efficacy and seizure-free rates, along with the side effect profile confirmed in C021 with a lower initial dose and slower up-titration every 2 weeks, cenobamate is a potentially important addition to currently available ASMS.

\section{Keywords}

Antiepileptic, anti-seizure medication, cenobamate, clinical studies, epilepsy, focal seizures, partial-onset seizures, seizures

Disclosures: Pavel Klein has been a consultant for Abbott, Eisai, Engage Therapeutics, and UCB Pharma; has participated in advisory boards for Aquestive, Eisai, and SK Life Science, Inc.; has been a speaker for Aquestive, Eisai, Sunovion, and UCB Pharma; is a member of a medical advisory board of Alliance and of a science advisory board of OB Pharmaceuticals; and has received research support from Eisai and Lundbeck. Louis Ferrari is an employee of SK Life Science, Inc. William E Rosenfeld is a

consultant/advisor for SK Life Science, Inc. has received honoraria for speaking from Eisai Greenwich Biosciences (GW Pharmaceuticals), SK Life Science, Inc., Sunovion, and UCB Pharma; and has received grant/research support from Greenwich Biosciences, Marinus, Medtronic, Neurelis, Ovid, SK Life Science, Inc., Takeda, UCB Pharma, and Upsher-Smith.

Acknowledgments: Medical writing assistance was provided by Dorothy McCoy, PharmD, BCPS, BCIDP, of MedVal Scientific Information Services, LLC (Princeton, NJ, USA) and was funded by SK Life Science, Inc. The views and opinions expressed are those of the author and do not necessarily reflect those of SK Life Science, Inc.

Review Process: Double-blind peer review.

Compliance with Ethics: This article involves a review of the literature and did not involve any studies with human or animal subjects performed by any of the authors.

Authorship: The named authors meet the International Committee of Medical Journal Editors (ICMJE) criteria for authorship of this manuscript, take responsibility for the integrity of the work as a whole, and have given final approval for the version to be published.

Access: This article is freely accessible at touchNEUROLOGY.com

(C) Touch Medical Media 2020

Received: June 3, 2020

Accepted: August 13, 2020

Published Online: December 23, 2020

Citation: US Neurology. 2020;16(2):87-97

Corresponding Author: Pavel Klein, Mid-Atlantic Epilepsy and Sleep Center, 6410 Rockledge Drive, Suite 610, Bethesda, MD 20817, USA.

E: KleinP@Epilepsydc.com

Support: No funding was received in the publication of this article.
Epilepsy is a very common neurological disease, affecting more than 50 million people worldwide and 3.4 million people in the USA. ${ }^{1-3}$ Focal seizures, formerly partial-onset seizures, are the most common type, making up $\geq 60 \%$ of cases. ${ }^{4-6}$ Patients with epilepsy have an increased risk of morbidity and mortality, a decreased quality of life and are more likely to have cognitive deficits, psychiatric disorders, and emotional and psychosocial difficulties. ${ }^{2.7}$ Pharmacotherapy with an anti-seizure medication (ASM)/antiepileptic drug, used as monotherapy or in combination, is the predominant treatment modality, 8,9 however, adverse effects and drug interactions may contribute to treatment failure. 10,11 Over $35 \%$ of patients continue to have seizures despite the use of various concomitant ASMs, and the likelihood of achieving seizure freedom decreases with each subsequent ASM regimen. 12,13 Patients with uncontrolled seizures may develop comorbidities such as memory and cognitive impairment, psychiatric disease, reproductive endocrine disease, and structural changes to the brain, and are at greater risk for sudden unexpected death in epilepsy and premature death., ${ }^{7,14}, 15$ This review focuses on cenobamate, a newly available ASM, and the clinical data regarding its efficacy and safety in uncontrolled focal (partial-onset) seizures.

\section{Cenobamate pharmacodynamics and pharmacokinetics}

Cenobamate is an oral ASM approved in the USA for the treatment of focal (partial-onset) seizures in adults. ${ }^{16,17}$ The precise mechanism by which cenobamate exerts its therapeutic effects in patients with focal seizures in unknown. ${ }^{17}$ Cenobamate has been demonstrated to reduce repetitive neuronal firing by inhibiting voltage-gated sodium currents, with preferential inhibition of the persistent current. 17,18 It is also a positive allosteric modulator of the $\gamma$-aminobutyric acid $\left(\mathrm{GABA}_{A}\right)$ ion channel, 17,19 binding to non-benzodiazepine $\mathrm{GABA}_{\mathrm{A}}$ receptor binding sites. ${ }^{19}$ 
Following oral administration, $88 \%$ of cenobamate is absorbed. ${ }^{17}$ No significant alterations in pharmacokinetic (PK) parameters were seen following a high-fat meal (800-1,000 calories with $50 \%$ fat). ${ }^{17}$ Cenobamate may be taken with or without food at any time and should be swallowed whole with liquid. PK data demonstrated dose-proportional increases in $\mathrm{C}_{\max }$ and the area under the concentration-time curve (AUC) after multiple dosing. 17,20 The volume of distribution is $40-50 \mathrm{~L}$ with $60 \%$ plasma protein binding, the majority of which is to albumin. ${ }^{17}$ Cenobamate has a terminal half-life of 50-60 hours, which allows for once-daily dosing, with steady state reached at approximately 2 weeks. ${ }^{17,20}$ Cenobamate is metabolized via glucuronidation, oxidation, and hydrolysis in the liver to inactive metabolites. ${ }^{17,20,21}$ The only metabolite in the plasma accounts for $1.2 \%$ (standard deviation 0.67 ) of the parent drug. ${ }^{21}$ Cenobamate and its metabolites are primarily excreted in the urine (approximately $88 \%$ of the administered dose). ${ }^{17,21}$

After a single dose of cenobamate $200 \mathrm{mg}$, the AUC was 1.4- and 1.5-times higher in subjects with mild (creatinine clearance $\left[\mathrm{Cl}_{\mathrm{Cr}}\right] 60-<90 \mathrm{~mL} / \mathrm{min}$ ) and moderate $\left(\mathrm{Cl}_{\mathrm{Cr}} 30-<60 \mathrm{~mL} / \mathrm{min}\right)$ renal impairment. Cenobamate AUC was 2.1- and 2.3-times higher in patients with mild (Child-Pugh Class A, 5-6 points) and moderate (Child-Pugh Class B, 7-9 points) hepatic impairment after a single dose of cenobamate $200 \mathrm{mg}$. Given the metabolism of cenobamate in the liver and the excretion of cenobamate and its metabolites in the urine, caution and dose reductions may be needed in patients with renal and/or hepatic impairment. ${ }^{17}$ A lower dose should be considered in patients with $\mathrm{Cl}_{\mathrm{Cr}}<90 \mathrm{~mL} / \mathrm{min}$ and the maximum recommended dose with Child-Pugh Class A or B hepatic impairment is reduced by half to $200 \mathrm{mg}$ once daily. PK data are not available in patients on hemodialysis or patients with severe hepatic impairment. No clinically relevant differences in PK were seen based on age in adults ranging from 18-77 years old. Renal and hepatic impairment, cardiac or other concomitant diseases and concurrent drug therapy should be taken into account when selecting the dose for elderly patients, starting with doses in the lower end of the dosing range. ${ }^{17}$

\section{Preclinical and early studies}

Rodent models demonstrated that cenobamate protects against maximal electroshock-induced seizures and pentylenetetrazol/picrotoxin-induced clonic seizures. ${ }^{22}$ It exhibited activity in lithium/pilocarpine-induced status epilepticus, hippocampal kindled and psychomotor seizures, and in spike-and-wave discharges in the genetic absence epilepsy rat from Strasbourg model.22,23 Altogether, preclinical studies confirmed that cenobamate has broad-spectrum activity with effects in focal, generalized, and absence seizures. Central nervous system (CNS)-related adverse events, such as somnolence, dizziness and gait disturbances, and nausea were reported in phase I studies. ${ }^{22,24}$ A proof-of-principle phase lla study in patients with photosensitive epilepsy established that partial suppression of intermittent photic stimulation-induced photoparoxysmal response occurred with a $100 \mathrm{mg}$ cenobamate dose..$^{25}$

\section{Cenobamate efficacy}

Two clinical studies have evaluated the efficacy of adjunctive cenobamate for the treatment of uncontrolled focal (partial-onset) seizures in adults, the details of which are listed in Table 1. In the first study, C013 (ClinicalTrials.gov identifier: NCT01397968), adults with uncontrolled focal seizures, despite ongoing treatment with one to three ASMs, were randomized to cenobamate or placebo for 12 weeks, including 6-week titration and 6-week maintenance phases. A total of 222 patients were treated. The five most frequent concomitant ASMs in the cenobamate group were levetiracetam, lamotrigine, carbamazepine, lacosamide, and topiramate. Cenobamate was initiated at $50 \mathrm{mg}$ once daily, a higher starting dose than is currently recommended in the XCOPRI ${ }^{\circledR}$ (SK Life Science, Inc., Paramus, $\mathrm{NJ}$, USA) prescribing information, and titrated every other week by 50-mg increments to a target maintenance dose of $200 \mathrm{mg} /$ day. ${ }^{17,26}$

The primary endpoint was the median percent reduction in seizure frequency during the double-blind treatment period (titration and maintenance phases) in the intention-to-treat (ITT) population. The cenobamate group had a $55.6 \%$ median reduction in seizure frequency as compared with $21.5 \%$ in the placebo group $(p<0.0001)$ (Table 1). The difference between cenobamate- and placebo-treated patients remained significant when assessing the secondary endpoint of median percent reduction for each focal seizure subtype. The median percent reduction in seizure frequency for specific types of focal seizures are listed in Table 1. Patients receiving cenobamate had a 50\% responder rate during the double-blind period of $50.4 \%$, while placebo-treated patients had a responder rate of $22.2 \%(p<0.0001)$ (Table 1). Cenobamate-treated patients were four times more likely than placebo-treated patients to have a $\geq 50 \%$ responder rate (odds ratio 3.94; 95\% confidence interval [CI]: 2.14-7.24). A post-hoc analysis demonstrated that during the maintenance phase, there were significant differences in the $\geq 50 \%, \geq 75 \%, \geq 90 \%$, and $100 \%$ seizure reduction rates between cenobamate- and placebo-treated patients, with approximately $20 \%$ more cenobamate-treated patients than placebo-treated patients experiencing seizure freedom (Table 1) (data on file, SK Life Science). In a post-hoc analysis of the completer population, cenobamate-treated patients experienced a substantial median percent reduction in seizure frequency $(40.6 \%$ decrease from baseline versus $14.3 \%$ for placebo, $p=0.001$ ) during the first 4 weeks of the titration phase, while going from 50 to $100 \mathrm{mg} /$ day. Cenobamate-treated patients continued to have further reductions in seizure frequency over the course of the study. ${ }^{26}$

Following completion of the double-blind treatment period in C013, patients from certain study sites were given the option to continue therapy in an open-label extension (OLE). Of 158 patients who were eligible, 149 (94.3\%) entered the OLE (76 had received cenobamate and 73 had received placebo in the double-blind treatment period). All OLE participants were initated on cenobamate $100 \mathrm{mg} /$ day with subsequent dose increases of $50 \mathrm{mg}$ every 2 weeks as tolerated, with a maximum dose of $400 \mathrm{mg} / \mathrm{day}$ (with or without a taper of the double-blind study drug prior to open-label conversion in order to minimize the potential for triggering seizure exacerabations in patients with a clinically meaningful response [data on file, SK Life Science]). At the time of data cut-off, in April 2018, the median modal daily cenobamate dose was $200 \mathrm{mg} /$ day and the median duration of cenobamate exposure was 60.6 months. The retention rate was $71 \%$ after 1 year and $65 \%$ after 2 years. A total of 60 patients discontinued the OLE: 28 due to withdrawal by the patient, 14 due to adverse events, 4 were lost to follow-up, 1 due to pregnancy, and 13 reported "other" as the reason for discontinuing the OLE. ${ }^{27}$ No patients discontinued the OLE due to lack of efficacy (data on file, SK Life Science).

In a second, larger dose-response study, C017 (NCT01866111), 437 adults with uncontrolled focal (partial-onset) seizures, despite ongoing therapy with one to three ASMs, were randomized 1:1:1:1 to cenobamate, titrated to maintenance doses of 100,200 , or $400 \mathrm{mg} /$ day, or placebo for 18 weeks, including 6-week titration and 12-week maintenance phases. Levetiracetam, lamotrigine, valproate or valproic acid, carbamazepine and oxcarbazepine 
Table 1: Adjunctive cenobamate efficacy and safety data from clinical studies (data on file, SK Life Science)

\begin{tabular}{|c|c|c|c|}
\hline & $\begin{array}{l}\text { Chung SS, et al. } \\
\text { Study } 013 \text { (NCT01397968)26 }\end{array}$ & $\begin{array}{l}\text { Krauss GL, et al. } \\
\text { Study } 017 \text { (NCT01866111)28 }\end{array}$ & $\begin{array}{l}\text { Sperling MR, et al. } \\
\text { Study } 021 \text { (NCT02535091)33 }\end{array}$ \\
\hline Type of study & Phase II, R, DB, followed by OLE & Phase II, R, DB, DR, followed by OLE & Phase III, open-label \\
\hline Seizure type & Focal, uncontrolled* & Focal, uncontrolled ${ }^{\dagger}$ & Focal, uncontrolled ${ }^{\dagger}$ \\
\hline $\begin{array}{l}\text { Cenobamate } \\
\text { starting dose, } \\
\text { mg/day }\end{array}$ & 50 & $50^{\ddagger}$ & 12.5 \\
\hline Titration schedule & $\uparrow$ by $50 \mathrm{mg}$ every 2 weeks & $\begin{array}{l}\uparrow \text { by } 50 \mathrm{mg} \text { every week up to } 200 \mathrm{mg} \text {, then } \\
100 \mathrm{mg} / \text { week thereafter }{ }^{\ddagger}\end{array}$ & $\begin{array}{l}\uparrow \text { to } 25 \mathrm{mg} \text { for weeks } 3 \text { and } 4,50 \mathrm{mg} \text { for } \\
\text { weeks } 5 \text { and } 6 \text {, and then by } 50 \mathrm{mg} \text { every } \\
2 \text { weeks thereafter }\end{array}$ \\
\hline $\begin{array}{l}\text { Titration phase, } \\
\text { weeks }\end{array}$ & 6 & 6 & 12 \\
\hline $\begin{array}{l}\text { Cenobamate } \\
\text { target dose for } \\
\text { maintenance phase, } \\
\mathrm{mg} / \text { day }\end{array}$ & $200(n=113)$ & $100(n=108) ; 200(n=110) ; 400(n=111)$ & $\begin{array}{l}\text { 200; could be } \uparrow \text { to a maximum dose of } 400 \\
(n=1,339)\end{array}$ \\
\hline $\begin{array}{l}\text { Maintenance phase, } \\
\text { weeks }\end{array}$ & 6 & 12 & $\geq 40$ \\
\hline Comparator & Placebo $(n=109)$ & Placebo $(n=108)$ & N/A \\
\hline Inclusion criteria & $\begin{array}{l}\text { - Adults 18-65 years old } \\
\text { - Taking 1-3 concomitant ASMs at } \\
\text { stable doses } \\
\text { - EEG confirming the diagnosis of } \\
\text { focal epilepsy } \\
\text { - Prior CT or MRI } \\
\text { - } \geq 3 \text { focal seizures per month (baseline } \\
\text { period } 8 \text { weeks) } \\
\text { - No consecutive 21-day seizure-free interval }\end{array}$ & $\begin{array}{l}\text { - Adults } 18-70 \text { years old } \\
\text { - Taking 1-3 concomitant ASMs at } \\
\text { stable doses } \\
\text { - EEG confirming the diagnosis of } \\
\text { focal epilepsy } \\
\text { - Prior CT or MRI } \\
\text { - } \geq 3 \text { focal seizures per month (baseline } \\
\text { period } 8 \text { weeks), with } \geq 8 \text { focal seizures } \\
\text { during baseline } \\
\text { - } \text { No consecutive 25-day seizure-free interval }\end{array}$ & $\begin{array}{l}\text { - Adults 18-70 years old } \\
\text { - Taking 1-3 concomitant ASMs at } \\
\text { stable doses } \\
\text { - EEG confirming the diagnosis of } \\
\text { focal epilepsy } \\
\text { - Prior CT or MRI }\end{array}$ \\
\hline Exclusion criteria & $\begin{array}{l}\text { - Taking vigabatrin within the past year } \\
\text { - Taking felbamate for <18 continuous months } \\
\text { - Taking intermittent rescue benzodiazepines } \\
\text { more than once per month within the } \\
\text { - } \text { past month } \\
\text { - History of status epilepticus, alcoholism, } \\
\text { - History of }>2 \text { allergic reactions to prior ASMs } \\
\text { - History of } 1 \text { serious hypersensitivity reaction }\end{array}$ & $\begin{array}{l}\text { - Taking vigabatrin within the past year } \\
\text { - Taking felbamate for < } 18 \text { continuous months } \\
\text { - Taking intermittent rescue benzodiazepines } \\
\text { more than once per month within the } \\
\text { past month } \\
\text { - Taking diazepam, phenytoin, } \\
\text { or phenobarbital } \\
\text { - History of status epilepticus, alcoholism, } \\
\text { drug abuse, or psychiatric illness } \\
\text { - History of a serious drug-induced } \\
\text { hypersensitivity reaction or drug-related } \\
\text { rash requiring treatment in a hospital, ASM } \\
\text { drug-associated rash involving conjunctiva } \\
\text { or mucosae, or }>1 \text { maculopapular rash } \\
\text { requiring discontinuation }\end{array}$ & $\begin{array}{l}\text { - Taking vigabatrin or ezogabine within the } \\
\text { - } \text { past year } \\
\text { - Hising felbamate for < } 18 \text { continuous months } \\
\text { drug abuse, or psychiatric illness } \\
\text { - History of any drug-induced rash or } \\
\text { hypersensitivity reaction } \\
\text { - First-degree relatives with a serious } \\
\text { cutaneous, drug-induced adverse reaction }\end{array}$ \\
\hline $\begin{array}{l}\text { Mean time since } \\
\text { epilepsy diagnosis, } \\
\text { years }\end{array}$ & $\begin{array}{l}\text { - } 23.0 \text { placebo } \\
\text { - } 22.6 \text { cenobamate } 200 \mathrm{mg}\end{array}$ & $\begin{array}{l}\text { - } 23.0 \text { placebo } \\
\text { - } 25.5 \text { cenobamate } 100 \mathrm{mg} \\
\text { - } 22.8 \text { cenobamate } 200 \mathrm{mg} \\
\text { - } 24.4 \text { cenobamate } 400 \mathrm{mg}\end{array}$ & 22.9 \\
\hline $\begin{array}{l}\text { Median baseline } \\
\text { focal seizure } \\
\text { frequency } / 28 \text { days }\end{array}$ & $\begin{array}{l}\text { - } 5.5 \text { placebo } \\
\text { - } 7.5 \text { cenobamate } 200 \mathrm{mg}\end{array}$ & $\begin{array}{l}\text { - } 8.4 \text { placebo } \\
\text { - } 9.5 \text { cenobamate } 100 \mathrm{mg} \\
\text { - } 11.0 \text { cenobamate } 200 \mathrm{mg} \\
\text { - } 9.0 \text { cenobamate } 400 \mathrm{mg}\end{array}$ & N/A \\
\hline
\end{tabular}


Table 1: Continued

\begin{tabular}{|c|c|c|c|}
\hline & $\begin{array}{l}\text { Chung SS, et al. } \\
\text { Study } 013 \text { (NCT01397968)26 }\end{array}$ & $\begin{array}{l}\text { Krauss GL, et al. } \\
\text { Study } 017\left(\text { NCT01866111) }{ }^{28}\right.\end{array}$ & $\begin{array}{l}\text { Sperling MR, et al. } \\
\text { Study } 021 \text { (NCT02535091) }^{33}\end{array}$ \\
\hline Seizure types, \% & $\begin{array}{l}\text { - Focal aware motor } \\
-22.9 \% \text { placebo } \\
-27.4 \% \text { cenobamate } 200 \mathrm{mg} \\
\text { - Focal impaired awareness } \\
-84.0 \% \text { placebo } \\
-73.0 \% \text { cenobamate } 200 \mathrm{mg} \\
\text { - Focal to bilateral tonic-clonic } \\
-61.5 \% \text { placebo } \\
-64.6 \% \text { cenobamate } 200 \mathrm{mg}\end{array}$ & $\begin{array}{l}\text { - Focal aware motor } \\
\text { - } 20 \% \text { placebo } \\
-23 \% \text { cenobamate } 100 \mathrm{mg} \\
-23 \% \text { cenobamate } 200 \mathrm{mg} \\
-20 \% \text { cenobamate } 400 \mathrm{mg} \\
\text { - Focal impaired awareness } \\
-78 \% \text { placebo } \\
-82 \% \text { cenobamate } 100 \mathrm{mg} \\
-76 \% \text { cenobamate } 200 \mathrm{mg} \\
-79 \% \text { cenobamate } 400 \mathrm{mg} \\
\text { - Focal to bilateral tonic-clonic } \\
-56 \% \text { placebo } \\
-64 \% \text { cenobamate } 100 \mathrm{mg} \\
-55 \% \text { cenobamate } 200 \mathrm{mg} \\
-65 \% \text { cenobamate } 400 \mathrm{mg}\end{array}$ & $\begin{array}{l}\text { - Focal aware motor } 24.2 \% \\
\text { - Focal impaired awareness } 77.4 \% \\
\text { - Focal to bilateral tonic-clonic } 58.7 \%\end{array}$ \\
\hline $\begin{array}{l}\text { Top } 5 \text { concomitant } \\
\text { ASMs, } \%\end{array}$ & $\begin{array}{l}\text { - } \text { Levetiracetam } \\
\text { - } 48.6 \% \text { placebo } \\
\text { - } 45.1 \% \text { cenobamate } 200 \mathrm{mg} \\
\text { - } \text { Lamotrigine } \\
-31.2 \% \text { placebo } \\
\text { - } 36.3 \% \text { cenobamate } 200 \mathrm{mg} \\
\text { - } \text { Carbamazepine } \\
\text { - } 39.4 \% \text { placebo } \\
\text { - } 33.6 \% \text { cenobamate } 200 \mathrm{mg} \\
\text { - } \text { Lacosamide } \\
-19.3 \% \text { placebo } \\
\text { - } 23.9 \% \text { cenobamate } 200 \mathrm{mg} \\
\text { - } \text { Topiramate } \\
-19.3 \% \text { placebo } \\
-22.1 \% \text { cenobamate } 200 \mathrm{mg}\end{array}$ & $\begin{array}{l}\text { - Levetiracetam } \\
-38 \% \text { placebo } \\
-44 \% \text { cenobamate } 100 \mathrm{mg} \\
-44 \% \text { cenobamate } 200 \mathrm{mg} \\
-45 \% \text { cenobamate } 400 \mathrm{mg} \\
\text { - Lamotrigine } \\
-28 \% \text { placebo } \\
-41 \% \text { cenobamate } 100 \mathrm{mg} \\
-25 \% \text { cenobamate } 200 \mathrm{mg} \\
-32 \% \text { cenobamate } 400 \mathrm{mg} \\
\text { - valproate or valproic acid } \\
-28 \% \text { placebo } \\
-21 \% \text { cenobamate } 100 \mathrm{mg} \\
-26 \% \text { cenobamate } 200 \mathrm{mg} \\
-25 \% \text { cenobamate } 400 \mathrm{mg} \\
\text { - Carbamazepine } \\
-36 \% \text { placebo } \\
-27 \% \text { cenobamate } 100 \mathrm{mg} \\
-25 \% \text { cenobamate } 200 \mathrm{mg} \\
-23 \% \text { cenobamate } 400 \mathrm{mg} \\
\text { - } 0 x \text { xarbazepine } \\
-12 \% \text { placebo } \\
-14 \% \text { cenobamate } 100 \mathrm{mg} \\
-16 \% \text { cenobamate } 200 \mathrm{mg} \\
-17 \% \text { cenobamate } 400 \mathrm{mg}\end{array}$ & $\begin{array}{l}\text { - } \text { Levetiracetam } 39.1 \% \\
\text { - } \text { Lamotrigine } 33.3 \% \\
\text { - } \text { Valproic acid, all forms } 30.8 \% \\
\text { - } \text { Carbamazepine } 27.6 \% \\
\text { - } \text { Lacosamide } 24.2 \%\end{array}$ \\
\hline $\begin{array}{l}\text { Median \% seizure } \\
\text { reduction from } \\
\text { baseline }^{\S}\end{array}$ & $\begin{array}{l}\text { ITT population (primary endpoint) } \\
\text { - } 21.5 \% \downarrow \text { placebo } \\
\text { - } 55.6 \% \downarrow \text { cenobamate } 200 \mathrm{mg}(\mathrm{p}<0.0001)\end{array}$ & $\begin{array}{l}\text { mIT population (FDA primary endpoint) } \\
\text { - } 24.0 \% \downarrow \text { placebo } \\
\text { - } 35.5 \% \downarrow \text { cenobamate } 100 \mathrm{mg}(\mathrm{p}=0.0071) \\
\text { - } 55.0 \% \downarrow \text { cenobamate } 200 \mathrm{mg}(\mathrm{p}<0.0001) \\
\text { - } 55.0 \% \downarrow \text { cenobamate } 400 \mathrm{mg}(\mathrm{p}<0.0001)\end{array}$ & N/A \\
\hline $\begin{array}{l}\text { Responder rate, } \\
\% \text { of patients }\end{array}$ & $\begin{array}{l}\text { ITT population (secondary endpoint) } \\
\text { - } 22.2 \% \text { placebo } \\
\text { - } 50.4 \% \text { cenobamate } 200 \mathrm{mg}(\mathrm{p}<0.0001) \\
\text { Post-hoc analysis (maintenance phase) } \\
\text { - } 32.4 \% \text { placebo } \\
\text { - } 62.3 \% \text { cenobamate } 200 \mathrm{mg}(\mathrm{p}<0.0001)\end{array}$ & $\begin{array}{l}\text { mITT-M population (EMA primary endpoint) } \\
\text { - } 25.5 \% \text { placebo } \\
\text { - } 40.2 \% \text { cenobamate } 100 \mathrm{mg}(\mathrm{p}=0.0365) \\
\text { - } 56.1 \% \text { cenobamate } 200 \mathrm{mg}(\mathrm{p}<0.0001) \\
\text { - } 64.2 \% \text { cenobamate } 400 \mathrm{mg}(\mathrm{p}<0.0001)\end{array}$ & N/A \\
\hline $\begin{array}{l}100 \% \text { seizure } \\
\text { reduction during } \\
\text { maintenance phase, } \\
\% \text { of patients }\end{array}$ & $\begin{array}{l}\text { Post-hoc analysis } \\
\text { - } 8.8 \% \text { placebo } \\
\text { - } 28.3 \% \text { cenobamate } 200 \mathrm{mg}(\mathrm{p}=0.0001)\end{array}$ & $\begin{array}{l}\text { Secondary endpoint } \\
\text { - } 1.0 \% \text { placebo } \\
\text { - } 3.9 \% \text { cenobamate } 100 \mathrm{mg}(\mathrm{p}=0.3688) \\
\text { - } 11.2 \% \text { cenobamate } 200 \mathrm{mg}(\mathrm{p}=0.0022) \\
\text { - } 21.1 \% \text { cenobamate } 400 \mathrm{mg}(\mathrm{p}<0.0001)\end{array}$ & N/A \\
\hline
\end{tabular}


Table 1: Continued

\begin{tabular}{|c|c|c|c|}
\hline & $\begin{array}{l}\text { Chung SS, et al. } \\
\text { Study } 013 \text { (NCT01397968)26 }\end{array}$ & $\begin{array}{l}\text { Krauss GL, et al. } \\
\text { Study } 017 \text { (NCT01866111)28 }\end{array}$ & $\begin{array}{l}\text { Sperling MR, et al. } \\
\text { Study } 021 \text { (NCT02535091) }\end{array}$ \\
\hline $\begin{array}{l}\geq 90 \% \text { seizure } \\
\text { reduction during } \\
\text { maintenance phase, } \\
\% \text { of patients }\end{array}$ & $\begin{array}{l}\text { Post-hoc analysis } \\
\text { - } 8.8 \% \text { placebo } \\
\text { - } 34.0 \% \text { cenobamate } 200 \mathrm{mg}(\mathrm{p}<0.0001)\end{array}$ & $\begin{array}{l}\text { Secondary endpoint } \\
\text { - } 2.9 \% \text { placebo } \\
\text { - } 8.8 \% \text { cenobamate } 100 \mathrm{mg}(\mathrm{p}=0.1340) \\
\text { - } 17.3 \% \text { cenobamate } 200 \mathrm{mg}(\mathrm{p}=0.0007) \\
\text { - } 28.4 \% \text { cenobamate } 400 \mathrm{mg}(\mathrm{p}<0.0001)\end{array}$ & N/A \\
\hline $\begin{array}{l}\text { Median \% seizure } \\
\text { reduction by seizure } \\
\text { subtype from } \\
\text { baseline }\end{array}$ & 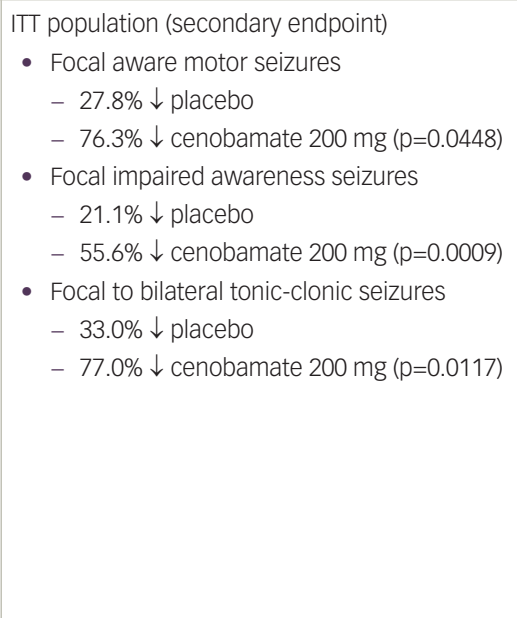 & \begin{tabular}{|l} 
mITT-M population (post-hoc analysis) \\
- Focal aware motor seizures \\
$-11.0 \% \uparrow$ placebo \\
$-49.0 \% \downarrow$ cenobamate $100 \mathrm{mg}(\mathrm{p}=0.0012)$ \\
$-62.0 \% \downarrow$ cenobamate $200 \mathrm{mg}(\mathrm{p}=0.0010)$ \\
$-69.0 \% \downarrow$ cenobamate $400 \mathrm{mg}(\mathrm{p}=0.0001)$ \\
- Focal impaired awareness seizures \\
$-29.0 \% \downarrow$ placebo \\
$-32.0 \% \downarrow$ cenobamate $100 \mathrm{mg}(\mathrm{p}=0.1022)$ \\
$-55.0 \% \downarrow$ cenobamate $200 \mathrm{mg}(\mathrm{p}=0.0003)$ \\
$-61.5 \% \downarrow$ cenobamate $400 \mathrm{mg}(\mathrm{p}<0.0001)$ \\
- Focal to bilateral tonic-clonic seizures \\
$-33.0 \% \downarrow$ placebo \\
$-51.0 \% \downarrow$ cenobamate $100 \mathrm{mg}(\mathrm{p}=0.2077)$ \\
$-92.0 \% \downarrow$ cenobamate $200 \mathrm{mg}(\mathrm{p}=0.0003)$ \\
$-83.0 \% \downarrow$ cenobamate $400 \mathrm{mg}(\mathrm{p}=0.0045)$
\end{tabular} & N/A \\
\hline $\begin{array}{l}\text { Most common } \\
\text { TEAEs, } \% \text { of patients } \\
\text { (occurring in } \geq 10 \% \\
\text { of patients with } \\
\text { any dose) }\end{array}$ & $\begin{array}{l}\text { - Somnolence } \\
-11.9 \% \text { placebo } \\
-22.1 \% \text { cenobamate } \\
\text { - Dizziness } \\
-16.5 \% \text { placebo } \\
-22.1 \% \text { cenobamate } \\
\text { - } \text { Headache } \\
-12.8 \% \text { placebo } \\
-12.4 \% \text { cenobamate } \\
\text { - } \text { Nausea } \\
-4.6 \% \text { placebo } \\
-11.5 \% \text { cenobamate } \\
\text { - Fatigue } \\
-6.4 \% \text { placebo } \\
-10.6 \% \text { cenobamate }\end{array}$ & 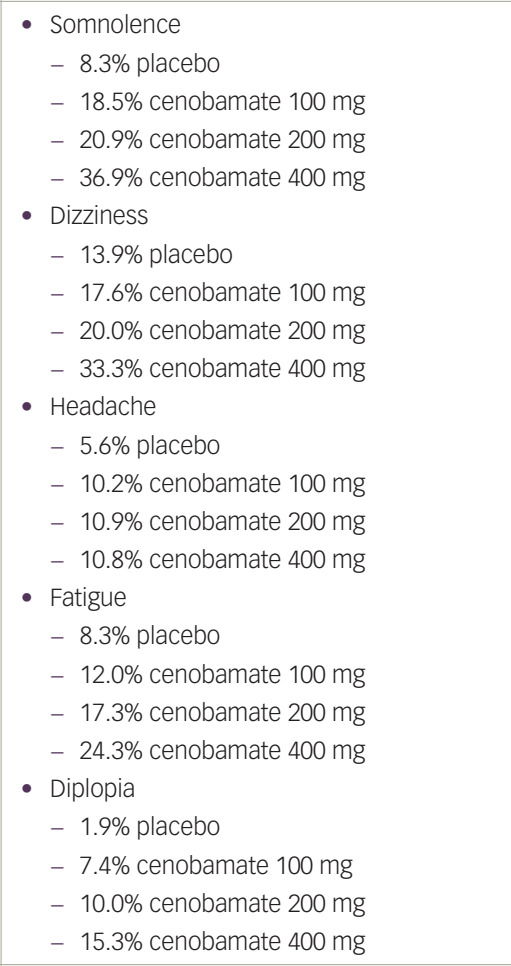 & $\begin{array}{l}\text { - } 28.1 \% \text { somnolence } \\
\text { - } 23.6 \% \text { dizziness } \\
\text { - } 16.6 \% \text { fatigue } \\
\text { - } 11.4 \% \text { headache }\end{array}$ \\
\hline $\begin{array}{l}\text { Serious TEAEs, } \\
\% \text { of patients }\end{array}$ & $\begin{array}{l}\text { - } 3.7 \% \text { placebo" } \\
\text { - } 1.8 \% \text { cenobamate }\end{array}$ & $\begin{array}{l}\text { - } 5.6 \% \text { placebo } \\
\text { - } 9.3 \% \text { cenobamate } 100 \mathrm{mg} \\
\text { - } 3.6 \% \text { cenobamate } 200 \mathrm{mg} \\
\text { - } 7.2 \% \text { cenobamate } 400 \mathrm{mg}\end{array}$ & $8.1 \%$ \\
\hline
\end{tabular}


Table 1: Continued

\begin{tabular}{|c|c|c|c|}
\hline & $\begin{array}{l}\text { Chung SS, et al. } \\
\text { Study } 013 \text { (NCT01397968)26 }\end{array}$ & $\begin{array}{l}\text { Krauss GL, et al. } \\
\text { Study } 017 \text { (NCT01866111)28 }\end{array}$ & $\begin{array}{l}\text { Sperling MR, et al. } \\
\text { Study } 021 \text { (NCT02535091) }^{33}\end{array}$ \\
\hline $\begin{array}{l}\text { Hypersensitivity } \\
\text { reactions in } \\
\text { cenobamate-treated } \\
\text { patients, n of } \\
\text { patients }\end{array}$ & $\begin{array}{l}1 \text { (reddening of palms and soles and itching } \\
\text { of ears) }\end{array}$ & $\begin{array}{l}3 \text { (1 non-serious pruritic rash with fever, } \\
1 \text { non-serious rash and facial swelling, } 1 \text { DRESS) }\end{array}$ & 1 \\
\hline DRESS, $n$ of patients & 0 & $\begin{array}{l}1 \text { (randomized to } 200 \mathrm{mg} \text { cenobamate with } \\
\text { weekly titration) }\end{array}$ & 0 \\
\hline $\begin{array}{l}\text { Deaths, } \mathrm{n} \text { of patients } \\
\text { (relationship to } \\
\text { study drug) }\end{array}$ & 1 (unrelated, occurred prior to randomization) & 0 & 4 (3 unrelated; 1 remotely related) \\
\hline
\end{tabular}

*Treatment-resistant ( $\geq 3$ seizures per month) despite treatment with one to three ASMS.

tSeizures despite treatment with at least one ASM within the past 2 years and taking stable doses of one to three concomitant ASMs.

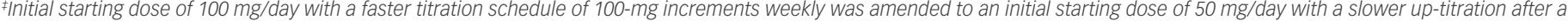
blinded review of the first nine patients.

${ }^{\S}$ Based on seizure frequency per 28 days.

Responder rate defined as $\geq 50 \%$ reduction in seizure frequency.

"Suicidal ideation, not considered a serious adverse event in this study, occurred in one patient in the cenobamate and one patient in the placebo group.

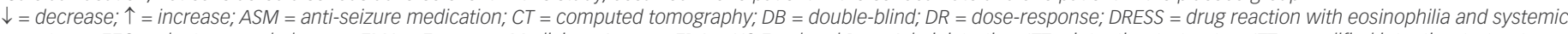
symptoms; $E E G$ = electroencephalogram; EMA = European Medicines Agency; FDA = US Food and Drug Administration; ITT = intention-to-treat; $m$-ITT = modified intention-to-treat; MITT-M = modified intention-to-treat-maintenance phase; $M R I=$ magnetic resonance imaging; $N / A=n o t$ applicable; $n=$ number; OLE = open-label extension; $R=$ randomized;

TEAE = treatment-emergent adverse event.

were the five most common concomitant ASMs across all groups. The initial cenobamate starting dose of the original titration schedule was $100 \mathrm{mg}$ once daily for 1 week with 100-mg increments each week until the target maintenance dose was reached. An amendment to the protocol was made after a blinded review of the first nine patients because of treatment-emergent adverse events (TEAES). ${ }^{28}$ Given the evidence that lower starting doses and slower up-titrations may improve tolerability, ${ }^{10,29}$ the starting dose for cenobamate was adjusted to $50 \mathrm{mg}$ once daily for 1 week with a slower up-titration of $50 \mathrm{mg}$ each week up to $200 \mathrm{mg} /$ day, and then increased by $100 \mathrm{mg}$ per week up to $400 \mathrm{mg} /$ day. ${ }^{28}$ Forty-six patients were enrolled in the study before the protocol was amended (data on file, SK Life Science). Of note, in both the original and amended protocol, the 100 and $50 \mathrm{mg} /$ day starting doses were higher and the weekly up-titrations were more rapid than is currently recommended. ${ }^{17}$

The primary endpoint for approval in the USA was median percent reduction in seizure frequency during the double-blind treatment period (titration and maintenance phases) in the modified ITT (MITT) population (all randomly assigned patients who had taken at least one dose of the study drug and had any post-baseline seizure data). There was a statistically significant median percent reduction in seizure frequency in all cenobamate dose groups versus placebo. A $24.0 \%$ reduction occurred in the placebo group versus a $35.5 \%$ reduction with cenobamate $100 \mathrm{mg} /$ day $(p=0.0071)$ and a $55.0 \%$ reduction with cenobamate $200 \mathrm{mg} /$ day $(p<0.0001)$ and $400 \mathrm{mg} /$ day $(p<0.0001)$ (Figure $1 a)$. The responder rate $(\geq 50 \%$ reduction in seizure frequency) in the mITT-maintenance (mITT-M) population (patients who completed the 6-week titration phase, took at least one dose of study medication, and had seizure data during the maintenance phase) was the primary endpoint for approval in the EU. The responder rate increased with increasing cenobamate doses (Figure 1b). ${ }^{28}$

Secondary endpoints included the median percent reduction in seizure frequency in the mITT-M population, the responder rate in the MITT population and the $\geq 75 \%$, $\geq 90 \%$, and $100 \%$ seizure reduction rates in the
mITT-M population. A dose-response was seen in the maintenance phase for the median percent reduction in seizure frequency: $27.0 \%$ decrease with placebo versus $41.5 \%$ decrease with cenobamate $100 \mathrm{mg} /$ day, $\mathrm{p}=0.0537 ; 56.5 \%$ decrease with cenobamate $200 \mathrm{mg} / \mathrm{day}, \mathrm{p}<0.0001$; and $63.0 \%$ decrease with cenobamate $400 \mathrm{mg} / \mathrm{day}, \mathrm{p}<0.0001$. The responder rates for the double-blind treatment period in the mITT population were analogous to those seen during the maintenance phase: $21.7 \%$ placebo versus $40.7 \%$ cenobamate $100 \mathrm{mg} / \mathrm{day}, \mathrm{p}=0.0032 ; 57.8 \%$ cenobamate 200 mg/day, $\mathrm{p}<0.0001$; and 60.4\% cenobamate 400 mg/day, $\mathrm{p}<0.0001$ (data on file, SK Life Science). Statistically significant seizure reduction rates of $\geq 75 \%, \geq 90 \%$, and $100 \%$ occurred in patients treated with cenobamate 200 and $400 \mathrm{mg} /$ day as compared to placebo-treated patients in the mITT-M population. Seizure freedom was achieved by $11.2 \%$ and $21.1 \%$ of patients treated with cenobamate 200 and $400 \mathrm{mg}$ /day compared with 1\% of placebo-treated patients (Figure 1C, Table 1).28

Additional analyses evaluated outcomes based on focal seizure subtypes during the maintenance phase. The median percent reduction in seizure frequency by seizure subtype was a secondary endpoint ( $p$-values assessed post-hoc). Statistically significant reductions in seizure frequency occurred in all seizure subtypes with cenobamate 200 and 400 mg/day in the mITT-M population (Table 1). Based on post-hoc analyses, the $\geq 50 \%$ responder rates for all seizure subtypes in all cenobamate dose groups ranged between $43.2 \%$ and $78.1 \%$, while in placebo-treated patients, the responder rates ranged between $11.8 \%$ and $48.8 \%$. Up to $30.0 \%$ of patients with focal aware (simple partial) motor seizures, $25.6 \%$ with focal impaired awareness (complex partial) seizures, and $52.8 \%$ with focal to bilateral tonic-clonic (secondarily generalized tonic-clonic) seizures experienced seizure freedom in the mITT-M population. ${ }^{28}$

In a post-hoc analysis of the mITT population in the double-blind treatment period there was an approximate $50 \%$ median percent reduction in seizure frequency during the first 4 weeks of treatment with all cenobamate dose groups (compared with $17 \%$ in the placebo group), while titrating from 


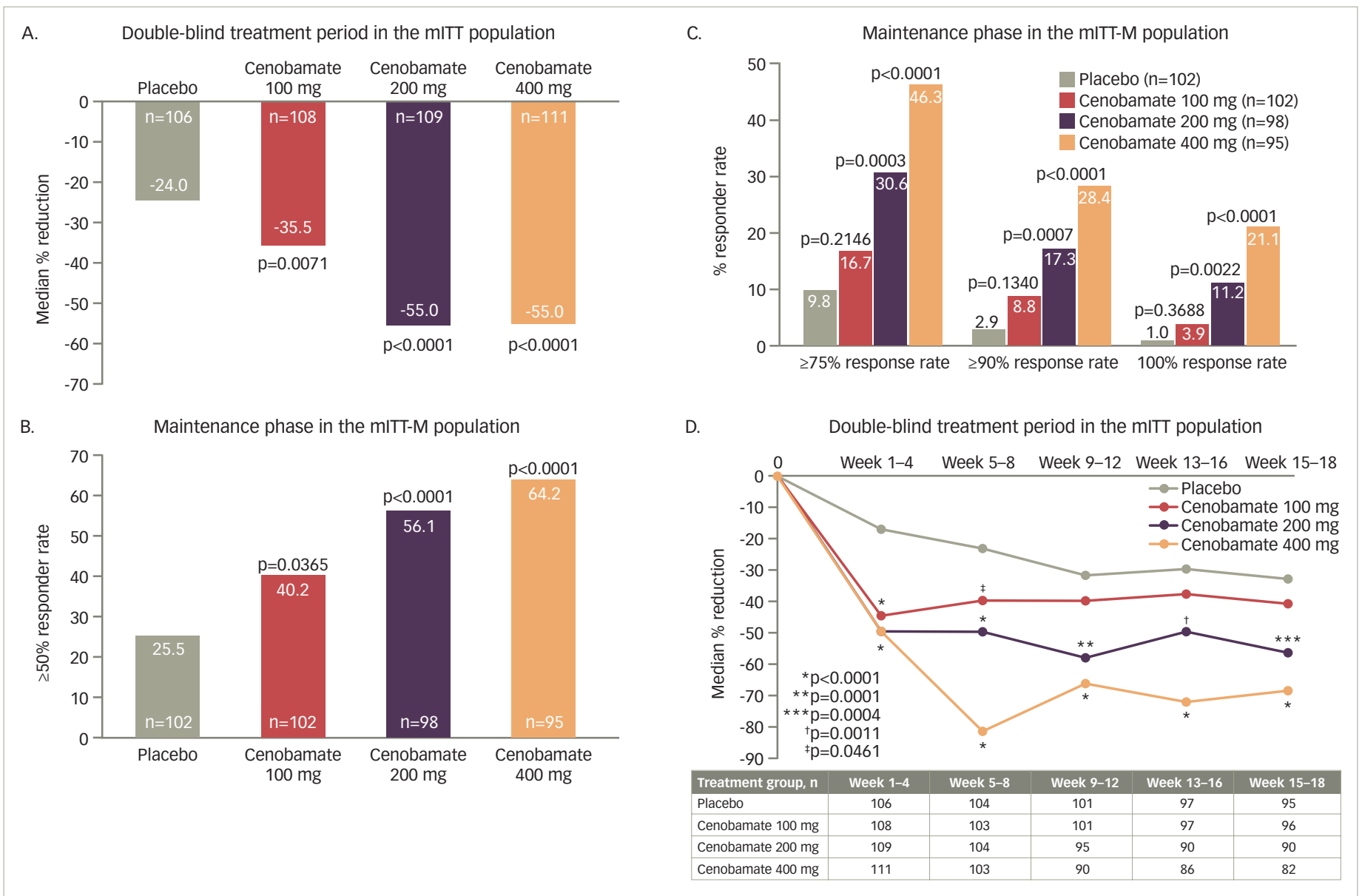

A. Median percent reduction in seizure frequency from baseline per 28 days in the double-blind treatment period in the modified intention-to-treat population; $B$. $\geq 50 \%$ responder rate during the maintenance phase in the mITT-M population; C. $\geq 75 \%, \geq 90 \%$, and $100 \%$ responder rates during the maintenance phase in the mITT-M population; and D. Median $\%$ reduction in seizure frequency from baseline per 28 days over time during the double-blind treatment period in the mITT population.

Double-blind treatment period included the 6-week titration phase and the 12-week maintenance phase.

mITT population included all patients randomly assigned to the treatment groups who took $\geq 1$ dose of study medication and had post-baseline seizure data.

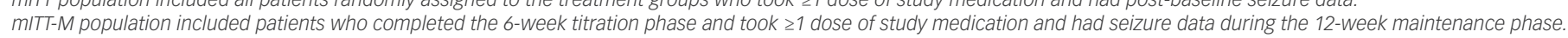

Weeks 13-16 and weeks 15-18 overlap, so that each interval was 4 weeks.

MITT = modified intention-to-treat; $m$-ITT- $M=$ modified intention-to-treat-maintenance.

Reproduced with permission from Krauss GL, et al. 2020.28

$50 \mathrm{mg} /$ day to the target dose of 100 or $200 \mathrm{mg} /$ day. The 200 and $400 \mathrm{mg} /$ day cenobamate groups continued to have further reductions over the course of the study (Figure 1d). The seizure-free rate in the first 4 weeks of the double-blind treatment period was approximately $6 \%$ in the cenobamate $100 \mathrm{mg} /$ day group and 11\% in both the cenobamate 200 and $400 \mathrm{mg} /$ day groups, compared with $1 \%$ of placebo-treated patients. The seizure-free rate reached about $7 \%, 20 \%$, and $28 \%$ in the cenobamate 100,200 , and $400 \mathrm{mg} /$ day groups, respectively, and $7 \%$ in the placebo group during study weeks 9-12 (Figure 2) (data on file, SK Life Science). ${ }^{28}$

Additional post-hoc analyses evaluated the effects of various baseline features on the median percent reduction in focal seizures and responder rates with adjunctive cenobamate in C017. Cenobamate-treated patients experienced clinically relevant reductions in median seizure frequency as compared with placebo, regardless of the severity of disease, which was defined by the number of baseline ASMs (1,2, or $>2)$, the number of median baseline siezures per 28 days $(\leq 9.5,>9.5)$, and the median baseline epilepsy duration in years $(\leq 23,>23)$. Cenobamate-treated patients also generally had higher responder $(\geq 50 \%$ ) and seizure freedom rates regardless of the severity of disease versus placebo-treated patients. ${ }^{30}$

After patients completed the entire double-blind treatment period in C017 they were given the option to enroll in the C017 OLE. A total of 355 out of 360 (98.6\%) patients who completed the double-blind treatment period elected to participate in the OLE (265 had received cenobamate and 90 had received placebo in the double-blind treatment period). Patients were converted to a target cenobamate dose of $300 \mathrm{mg} /$ day over a 2-week blinded period. At the time of data cut-off, in April 2018, patients received a median modal dose of $300 \mathrm{mg} /$ day of cenobamate for a median of 40 months. After 1 and 2 years, the retention of patients in the OLE was $80 \%$ and $70 \%$, respectively. A total of 125 patients discontinued the OLE, including 55 patients due to lack of efficacy, 24 due to adverse events, 23 due to withdrawal by the patient, 7 were lost to follow-up, 5 had died, 2 had protocol violations, and 9 reported "other" as the reason for discontinuing the OLE. The median reduction in seizure frequency was $65.4 \%$ in all OLE patients in months $1-6$. Patients treated with cenobamate during the double-blind period who entered the 
Figure 2: Study C017 seizure-free rate over time during the double-blind treatment period in the modified intention-to-treat population

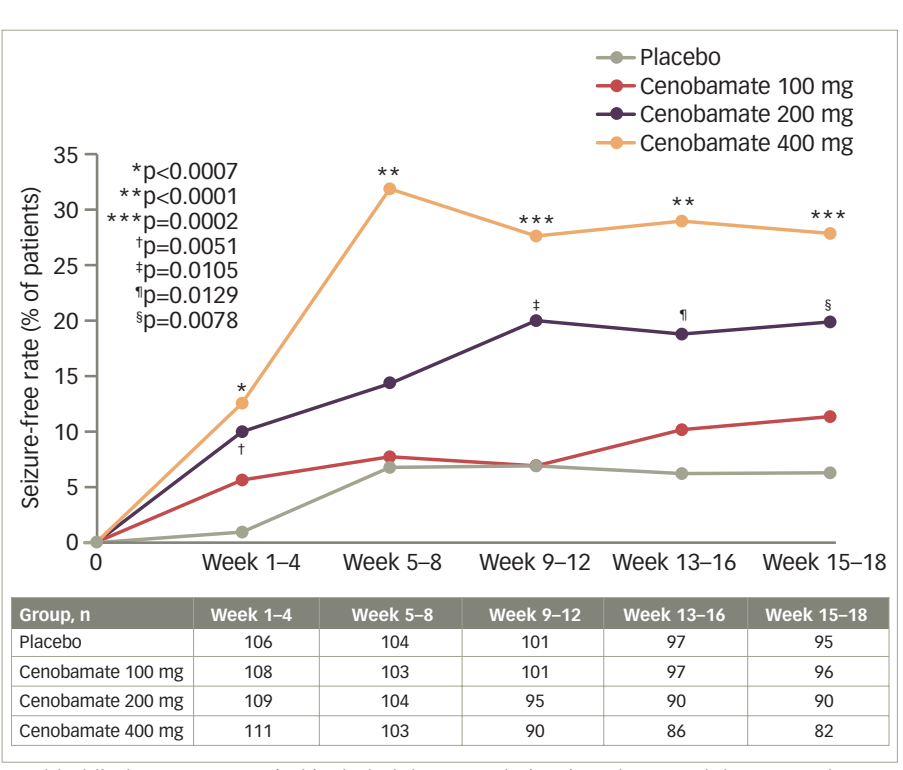

Double-blind treatment period included the 6-week titration phase and the 12-week maintenance phase.

Modified intention-to-treat population included all patients randomly assigned to the treatment groups who took $\geq 1$ dose of study medication and had post-baseline seizure data. Weeks 13-16 and weeks 15-18 overlap, so that each interval was 4 weeks.

Reproduced with permission from Krauss GL, et al. ${ }^{28}$

OLE and continued to receive cenobamate, and patients treated with placebo during the double-blind period who entered the OLE and were switched to cenobamate, had similar median reductions in seizure frequency $(65.7 \%$ and $63.0 \%$, respectively) at this time point. Further median reductions occurred over time in all groups. Similarly, $\geq 50 \%$ responder rates increased over time in all OLE patients, reaching $74.9 \%$ in months $25-30$. Seizure freedom was achieved in $20.2 \%$ (45/223) of evaluable OLE patients at 25-30 months. Among the 45 patients who were seizure-free at months $25-30$, the median duration of seizure freedom achieved during the entire OLE was 33.2 months (range 13.2-50.4 months). ${ }^{31}$

Cenobamate 100,200 , and $400 \mathrm{mg} /$ day produced clinically relevant median percent reductions in seizure frequency in adults with uncontrolled focal epilepsy. ${ }^{26,28}$ During the double-blind treatment period the median percent reduction in seizure frequency in both studies ranged from 35.5\% (cenobamate $100 \mathrm{mg} /$ day in C017) to approximately 55\% (cenobamate 200 mg/day in C013 and cenobamate 200 and 400 mg/day in C017), with greater reductions seen with cenobamate 200 and 400 mg/day. ${ }^{26,28}$ During the maintenance phase in C017, the reduction in seizure frequency reached $63.0 \%$ with cenobamate $400 \mathrm{mg} /$ day ${ }^{28}$ Approximately $\geq 50 \%$ of patients treated with cenobamate $\geq 200 \mathrm{mg} /$ day in both studies had a $\geq 50 \%$ reduction in seizure frequency in the double-blind period, with the responder rate in C017 ranging from 40.2\% (cenobamate $100 \mathrm{mg} / \mathrm{day}$ ) to $64.2 \%$ (cenobamate $400 \mathrm{mg} /$ day) in the maintenance phase. ${ }^{26,28}$ During the maintenance phase in C013 (cenobamate $200 \mathrm{mg} /$ day) and C017 (cenobamate $400 \mathrm{mg} / \mathrm{day}$ ), the difference between cenobamate- and placebo-treated patients in achieving seizure freedom was $20 \%{ }^{26,28}$ Cenobamate decreased seizure frequency in all focal seizure subtypes. ${ }^{26,28}$ In both studies a 40-50\% median reduction in seizure frequency occurred in the first 4 weeks of titration from a dose of $50 \mathrm{mg} /$ day to the target maintenance dose, and further reductions occurred in patients treated with cenobamate 200 (C013, C017) and $400 \mathrm{mg}$ /day (C017) over time..$^{26,28} \mathrm{In}$ particular for C017, patients achieved seizure freedom in the first 4 weeks of cenobamate treatment as the dose was being titrated from the starting dose of $50 \mathrm{mg} /$ day to target maintenance doses of 100 or $200 \mathrm{mg} /$ day, with an increase in the seizure-free rate over time in all cenobamate dose groups. ${ }^{28}$ The C017 OLE confirmed that seizure reduction was maintained over a prolonged duration of time. ${ }^{31}$

\section{Cenobamate safety}

Three clinical studies (C013, C017, C021) have evaluated the safety of cenobamate. Somnolence, dizziness, headache, nausea, and fatigue occurred in $\geq 10 \%$ of cenobamate-treated patients in C013 (Table 1). The majority of TEAEs (74.3\%) were mild or moderate in severity. Five patients discontinued cenobamate due to eight TEAEs (tachycardia, abdominal pain, gastroesophageal reflux, drug hypersensitivity, nystagmus, aggression, depression, dyspnea) (data on file, SK Life Science). No cases of drug reaction with eosinophilia and systemic symptoms (DRESS) occurred. ${ }^{26}$ Similarly, in C017 the TEAEs that occurred in $\geq 10 \%$ of cenobamate-treated patients were CNS-related (somnolence, dizziness, headache, fatigue) along with diplopia (Table 1). Mild or moderate TEAEs occurred in $54.6 \%$ of patients treated with cenobamate $100 \mathrm{mg} /$ day, 66.4\% of patients treated with cenobamate $200 \mathrm{mg} /$ day, and $73.9 \%$ of patients treated with cenobamate $400 \mathrm{mg} /$ day. TEAEs that led to treatment discontinuation in cenobamate-treated patients occurred in $10.2 \%$ of patients who received $100 \mathrm{mg} /$ day, $13.6 \%$ of patients who received $200 \mathrm{mg} /$ day, and $19.8 \%$ of patients who received $400 \mathrm{mg} /$ day. The TEAES that led to $\geq 1$ patient discontinuing in any group included ataxia, somnolence, dizziness, nystagmus, and vertigo. There was one case of DRESS. ${ }^{28} \mathrm{~A}$ post-hoc analysis of the data from $\mathrm{C} 013$ and C017 found that CNS-related events and TEAEs that led to treatment discontinuation occurred most often during the titration phase. ${ }^{32}$ Of note, dose adjustment of concomitant ASMs was not allowed during the double-blind treatment periods in $\mathrm{C} 013$ and C017. ${ }^{26,28}$

C021 (NCT02535091) is a phase III, open-label, ongoing long-term (12-month) safety study in adults with uncontrolled focal (partial-onset) seizures. $^{33}$ Unlike the efficacy studies, patients taking concomitant phenytoin and phenobarbital were included in C021 for a PK analysis to evaluate the impact of cenobamate on their plasma concentrations (reviewed below in the drug-drug interactions section). As prior studies identified three cases of DRESS (two in phase I studies with one fatality, one in C017) in patients who used a titration schedule faster than every 2 weeks and a starting dose of $\geq 50 \mathrm{mg} /$ day of cenobamate (data on file, SK Life Science), ${ }^{28,33}$ C021 specifically utilized a titration schedule with a lower starting dose $(12.5 \mathrm{mg} /$ day) and a slower titration rate (every 2 weeks) to minimize the risk of DRESS. ${ }^{33,34}$ Cenobamate was titrated over 12 weeks to an initial target dose of $200 \mathrm{mg}$ /day $(12.5,25,50,100,150$, and $200 \mathrm{mg}$ /day at 2-week intervals), with increases to a maximum of $400 \mathrm{mg} /$ day (using biweekly increments of $50 \mathrm{mg} /$ day) permitted if clinically warranted. Cenobamate exposure was $\geq 6$ months for $82.9 \%(1,110 / 1,339$ patients) of the patients at the time of the interim analysis. Levetiracetam, lamotrigine, all forms of valproic acid, carbamazepine, and lacosamide made up the five most common concomitant ASMs, with only $8.5 \%$ and $3.8 \%$ of patients taking concomitant phenytoin and phenobarbital, respectively. TEAEs that occurred in $\geq 10 \%$ of patients were somnolence, dizziness, fatigue, and headache (Table 1). Most (77.8\%) of the TEAEs were 
mild or moderate in severity. TEAEs related to nervous system disorders (3.4\%) and skin and subcutaneous tissue disorders (3.3\%) were the most common TEAEs leading to cenobamate discontinuation. There were no cases of DRESS. ${ }^{33}$

The most frequent TEAEs during the double-blind periods in $\mathrm{C} 013$ and C017, and in the open-label C021 study, were CNS-related events, with higher rates reported with cenobamate $400 \mathrm{mg} /$ day as compared with 100 and 200 mg/day in C017 (Table 1). The majority of TEAEs in cenobamate-treated patients were mild or moderate and discontinuation rates due to TEAEs were low. Of these three clinical studies, one case of DRESS occurred in C017 during the original protocol, which started with an initial dose of $100 \mathrm{mg} /$ day and titrated up by $100 \mathrm{mg}$ weekly (the protocol was later amended to decrease the starting dose to $50 \mathrm{mg}$ /day and use a slower titration to improve tolerability) (Table 1). No cases of DRESS occurred in C013 or C021, where initial doses of $50 \mathrm{mg}$ /day (not the recommended starting dose) and $12.5 \mathrm{mg}$ /day were used, respectively, coupled with titration every 2 weeks.

\section{Drug-drug interactions}

Cenobamate exposure is marginally impacted by other ASMS. co-administration with phenobarbital, carbamazepine, or valproate led to minimal change in cenobamate multiple-dose exposure. Cenobamate $\mathrm{C}_{\max }$ and AUC decreased by $27 \%$ and $28 \%$, respectively, when cenobamate was given concurrently with phenytoin. ${ }^{17}$

In vivo drug-drug interaction studies demonstrated that cenobamate is an inducer of CYP2B6 and CYP3A4 and inhibitor of CYP2C19. ${ }^{17,35,36}$ A CYP450 probe study found that cenobamate induces CYP2B6 (bupropion probe) and has dose-dependent CYP3A4 (midazolam probe) induction, with $200 \mathrm{mg}$ of cenobamate having a greater impact on midazolam $\mathrm{C}_{\max }$ and AUC than $100 \mathrm{mg}$ of cenobamate. The probe study also established that cenobamate inhibits CYP2C19 (omeprazole probe) and has no effect on CYP2C9 (warfarin probe). ${ }^{36}$

Certain ASMs may need to be dose adjusted when used concomitantly with cenobamate. Based on population PK analyses, concurrent administration of cenobamate is expected to decrease lamotrigine plasma concentrations by $21-52 \%$, suggesting an increase in the lamotrigine dose may need to be considered. ${ }^{17}$ However, data from a subset of study sites in the C021 study indicated that the mean dose of lamotrigine was actually reduced by investigators due to pharmacodynamic considerations when used concomitantly with cenobamate (data on file, SK Life Science). Mean carbamazepine $\mathrm{C}_{\max }$ and AUC were both shown to decrease by $23 \%$ with co-administration of cenobamate due to CYP3A4 induction. ${ }^{11,35}$ In contrast, cenobamate's inhibition of CYP2C19 resulted in increased plasma concentrations of phenytoin and phenobarbital when these medications were co-administered with cenobamate. ${ }^{11,35}$ Cenobamate increased mean phenytoin $\mathrm{C}_{\max }$ and $\mathrm{AUC}$ by $70 \%$ and $84 \%$, respectively, and mean phenobarbital $\mathrm{C}_{\max }$ and $\mathrm{AUC}$ by $34 \%$ and $37 \% .{ }^{17}$ Increased concentrations of the active metabolite of clobazam, $\mathrm{N}$-desmethylclobazam, are also expected to occur due to CYP2C19 inhibition. ${ }^{11,17}$ Dose reductions of these three ASMs may be warranted when they are taken with cenobamate to minimize the risk of their adverse effects. For phenytoin, doses need to be titrated down by as much as $50 \%$ as cenobamate is being titrated up. ${ }^{17}$ A PK evaluation from the C021 study suggested that reduction of the phenytoin dose may need to begin when cenobamate is titrated from 25 to $50 \mathrm{mg} /$ day. In this study, $43.4 \%$ and $29.7 \%$ of patients taking phenytoin and phenobarbital had their doses decreased. Periodic reductions in phenytoin and phenobarbital doses of 25-33\% (by no more than up to two-thirds the total baseline dose), based on adverse events, plasma concentrations, and/or clinical discretion, resulted in relatively stable mean plasma concentrations of both drugs during cenobamate titration. ${ }^{33}$

For non-ASM medications, doses of CYP2B6 substrates (e.g., efavirenz ${ }^{37}$ and CYP3A substrates (e.g., benzodiazepines, protease inhibitors, statins) ${ }^{17,37}$ may need to be increased to maintain their efficacy as co-administration with cenobamate may decrease their plasma concentrations. ${ }^{17}$ Doses of CYP2C19 substrates (e.g., omeprazole and voriconazole) ${ }^{37}$ may need to be decreased to reduce the risk of adverse effects when they are used with cenobamate as concomitant administration increases their plasma concentrations. ${ }^{17}$ Women taking oral contraceptives and cenobamate simultaneously should use additional or alternative non-hormonal birth control as cenobamate may decrease plasma concentrations of contraceptive steroids. ${ }^{17}$

\section{Cenobamate dosing}

Based on the c021 study, the recommended dosing in the XCOPRI prescribing information reflects the initial dose and titration schedule from that study. Increasing daily doses of cenobamate are administered $(12.5,25,50,100,150$, and $200 \mathrm{mg})$ at 2-week intervals. Further dose escalation may be warranted after at least 2 weeks of treatment with $200 \mathrm{mg} /$ day, based on clinical response and tolerability, in which case cenobamate can be increased by $50 \mathrm{mg}$ once daily every 2 weeks, up to a maximum maintenance dose of $400 \mathrm{mg} /$ day (Figure 3). When discontinuing cenobamate the dose should be down-titrated over a period of $\geq 2$ weeks; if safety concerns require abrupt withdrawal, the potential risks of abrupt discontinuation may be mitigated by cenobamate's long half-life. ${ }^{17}$

\section{Overall summary}

Two pivotal studies, in similar patient populations and using different cenobamate titration schedules, have demonstrated the efficacy of adjunctive cenobamate in adults with uncontrolled focal epilepsy despite treatment with one to three ASMs at doses of 100, 200, and $400 \mathrm{mg} /$ day. Cenobamate reduced the frequency of focal (partial-onset) seizures by approximately 55\% from baseline, with some patients experiencing greater reductions with higher doses. Reductions in seizure frequency may occur with doses as low as $50 \mathrm{mg} /$ day during titration, prior to patients reaching the cenobamate maintenance dose of $\geq 200 \mathrm{mg} /$ day, up to a maximum dose of $400 \mathrm{mg} /$ day. The seizure freedom rates are encouraging and higher than previously reported with other adjunctive ASMs in uncontrolled focal epilepsy, with up to $20 \%$ more cenobamate-treated patients, varying by dose, compared with placebo-treated patients achieving seizure freedom with adjunctive cenobamate. ${ }^{38,39}$ However, comparisons to results from other studies have limitations due to differences in study designs and inconsistent methods for analyzing seizure freedom. In line with other randomized, placebo-controlled studies of adjunctive ASMs, a number of placebo-treated patients in the $\mathrm{C013}$ and $\mathrm{C017}$ studies had reductions in seizure frequency or achieved seizure freedom..$^{39-41}$ This phenomenon of "placebo response" is not exclusive to the adjunctive cenobamate clinical studies and has been documented in other adjunctive ASM studies, whereby patients in both the placebo and study-drug groups are taking background ASMs, to be approximately $20 \%$ seizure free. ${ }^{42}$ The "placebo response" is suggested to be influenced by study design, patient population, patient expectations, duration of the study, and patient-investigator interaction 


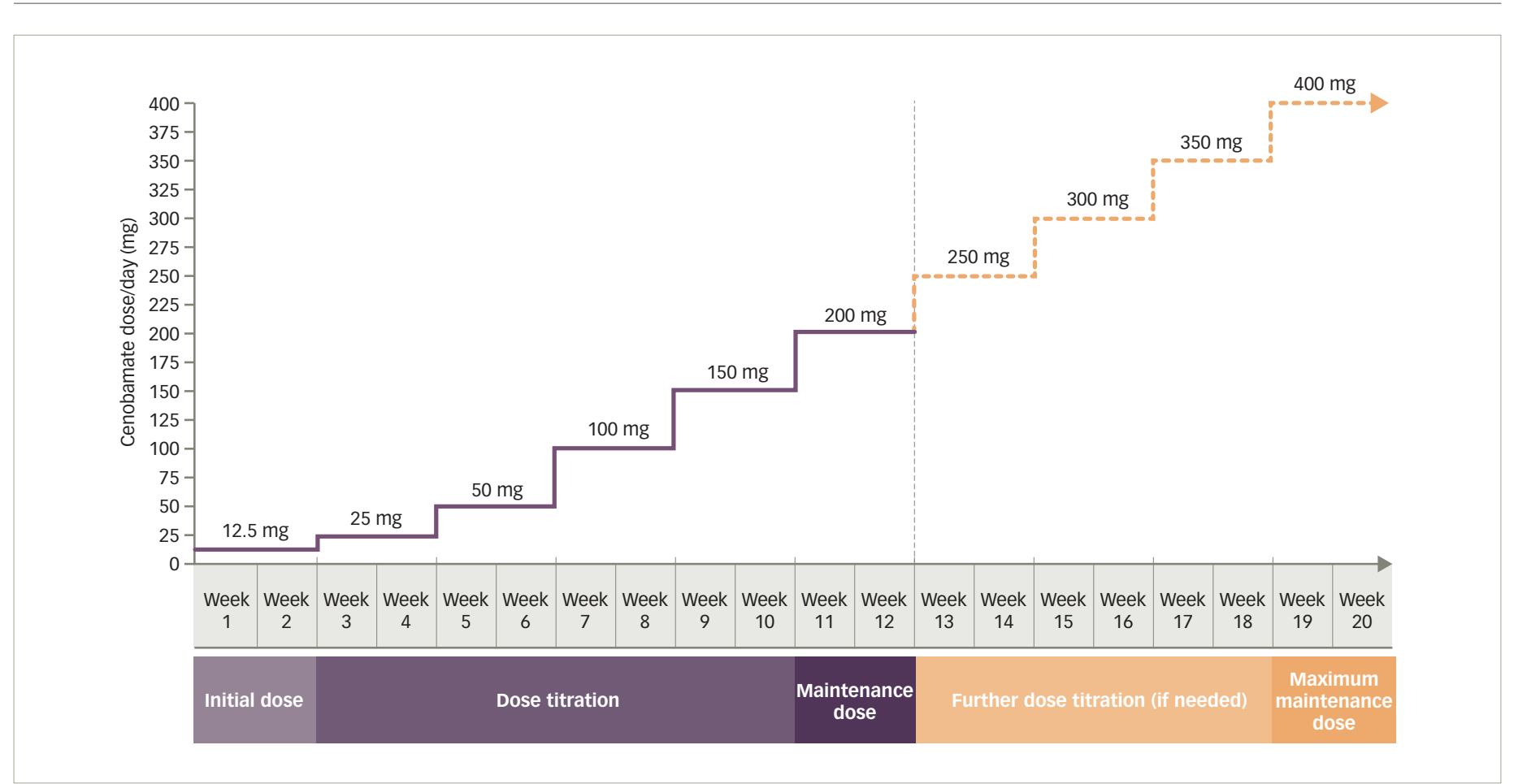

The dose may be increased up to a maximum of $400 \mathrm{mg}$ orally once daily based on clinical response and tolerability.

Dosing recommendations for patients with normal renal and hepatic function. Dosage reduction may be considered in patients with mild to moderate and severe renal impairment.

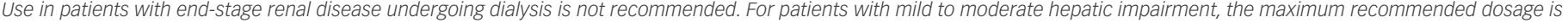
$200 \mathrm{mg}$ once daily. Use is not recommended in patients with severe hepatic impairment.

during clinical trials. ${ }^{42,43}$ Cenobamate is generally safe, with CNS-related side effects being the most commonly reported TEAEs. Although three cases of DRESS have been reported, no cases were reported with the dosing regimen used in the c021 study, which is the regimen recommended in the XCOPRI prescribing information (i.e., starting with a low dose of $12.5 \mathrm{mg} /$ day and increasing the dose slowly every 2 weeks). Altogether, the data suggest that adjunctive cenobamate shows great promise as a treatment option for patients with uncontrolled focal epilepsy.

\section{Article highlights}

- The efficacy of cenobamate, an oral anti-seizure medication (ASM) approved in the USA for the treatment of focal (partial-onset) seizures in adults, was studied in two randomized, double-blind (titration plus maintenance phase), clinical studies (C013 and c017) in patients with uncontrolled focal epilepsy

- In $\mathrm{C} 013$, adjunctive cenobamate at a dose of $200 \mathrm{mg} /$ day produced a median percent reduction in seizure frequency of $55.6 \%$ in the double-blind treatment period, with $a \geq 50 \%$ responder rate of $62.3 \%$ in the maintenance phase of therapy

- At doses of 100,200 , and $400 \mathrm{mg} /$ day in C017, the median percent reduction in seizure frequency in the double-blind treatment period was $35.5 \%$, $55.0 \%$, and $55.0 \%$, respectively; and the $\geq 50 \%$ responder rate during the maintenance phase of therapy was $40.2 \%, 56.1 \%$, and $64.2 \%$, respectively

- In C013 and C017, 28.3\% (200 mg/day in C013) and 21.1\% (400 mg/day in C017) of cenobamate-treated patients achieved zero seizures (seizure freedom) during the maintenance phase of therapy compared to 8.8\% (C013) and 1.0\% (C017) of placebo-treated patients

- The most commonly reported treatment-emergent adverse events across the two clinical studies (C013, C017) and a long-term open-label safety study (C021) were central nervous system-related (somnolence, dizziness, fatigue)

- Although three cases of drug reaction with eosinophilia and systemic symptoms (DRESS) have been reported with cenobamate, no cases were reported with the low starting dose (12.5 mg/day) and slow titration (every 2 weeks) dosing regimen used in the c021 safety study

- While this finding from C021 does not establish that the risk of DRESS is prevented by slower titration, per the prescribing information, XCOPRI ${ }^{\circledR}$ (SK Life Science, Inc., Paramus, NJ, USA) should be initiated at a dose of $12.5 \mathrm{mg} /$ day for 2 weeks, with increasing daily doses (25, 50, 100, 150, and $200 \mathrm{mg}$ ) at 2-week intervals; further escalation up to a maximum of $400 \mathrm{mg} /$ day may be warranted based on clinical response and tolerability (50-mg increments at 2-week intervals after 2 weeks of therapy with $200 \mathrm{mg} /$ day)

- While cenobamate exposure is marginally impacted by other ASMs, the dose of some ASMs may need to be adjusted (carbamazepine, clobazam, lamotrigine, phenobarbital, and phenytoin) when used concomitantly with cenobamate 
1. Epilepsy Foundation. Facts about seizures and epilepsy. 2019 Available at: www.epilepsy.com/learn/about-epilepsy-basics/ facts-about-seizures-and-epilepsy (accessed August 14, 2020).

2. World Health Organization. Epilepsy: a public health imperative. 2019. Available at: www.who.int/mental_health/neurology/ epilepsy/report 2019/en/ (accessed August 14, 2020).

3. Zack MM, Kobau R. National and state estimates of the numbers of adults and children with active epilepsy - United States, 2015 MMWR Morb Mortal Wkly Rep. 2017:66:821-5.

4. Fisher RS, Cross JH, French JA, et al. Operational classification of seizure types by the International League Against Epilepsy: position paper of the ILAE Commission for Classification and Terminology. Epilepsia. 2017:58:522-30.

5. Wirrell EC, Grossardt BR, Wong-Kisiel LC, Nickels KC. Incidence and classification of new-onset epilepsy and epilepsy syndromes in children in Olmsted County, Minnesota from 1980 to 2004: a population-based study. Epilepsy Res. 2011;95:110-8.

6. Hauser WA, Annegers JF, Rocca WA. Descriptive epidemiology of epilepsy: contributions of population-based studies from Rochester, Minnesota. Mayo Clin Proc. 1996;71:576-86.

7. Laxer KD, Trinka E, Hirsch $\mathrm{L}$, et al. The consequences of refractory epilepsy and its treatment. Epilepsy Behav. 2014;37:59-70.

8. Perucca P, Scheffer IE, Kiley M. The management of epilepsy in children and adults. Med J Aust. 2018;208:226-33.

9. Golyala A, Kwan P. Drug development for refractory epilepsy: the past 25 years and beyond. Seizure. 2017;44:147-56

10. Perucca P, Gilliam FG. Adverse effects of antiepileptic drugs. Lancet Neurol. 2012;11:792-802.

11. Zaccara G, Perucca E. Interactions between antiepileptic drugs, and between antiepileptic drugs and other drugs. Epileptic Disord. 2014;16:409-31.

12. Chen Z, Brodie MJ, Liew D, Kwan P. Treatment outcomes in patients with newly diagnosed epilepsy treated with established and new antiepileptic drugs: a 30-year longitudinal cohort study. JAMA Neurol. 2018;75:279-86.

13. Tian N, Boring M, Kobau R, Zack MM, Croft JB. Active epilepsy and seizure control in adults - United States, 2013 and 2015. MMWR Morb Mortal Wkly Rep. 2018;67:437-42.

14. Nevalainen $\mathrm{O}$, Ansakorpi $\mathrm{H}$, Simola M, et al. Epilepsy-related clinical characteristics and mortality: a systematic review and meta-analysis. Neurology. 2014;83:1968-77.

15. Atif M, Sarwar MR, Scahill S. The relationship between epilepsy and sexual dysfunction: a review of the literature. Springerplus. 2016;5:2070

16. US Food and Drug Administration (FDA). FDA approves new treatment for adults with partial-onset seizures. 2019. Available at: www.fda.gov/news-events/press-announcements/fda-approvesnew-treatment-adults-partial-onset-seizures (accessed August 14, 2020).
17. XCOPRI ${ }^{\oplus}$ (cenobamate tablets), for oral use, CV [prescribing information]. Paramus, NJ: SK Life Science, Inc.; August 2020

18. Nakamura M, Cho JH, Shin HS, Jang IS. Effects of cenobamate (YKP3089), a newly developed anti-epileptic drug, on voltage-gated sodium channels in rat hippocampal CA3 neurons. Eur J Pharmacol. 2019:855:175-82.

19. Sharma R, Nakamura $M$, Neupane $C$, et al. Positive allosteric modulation of $\mathrm{GABA}_{\mathrm{A}}$ receptors by a novel antiepileptic drug cenobamate. Eur I Pharmacol. 2020:879:173117.

20. Vernillet $V$, Greene SA, Kamin M. Pharmacokinetics of cenobamate: results from single and multiple oral ascending-dose studies in healthy subjects. Clin Pharmacol Drug Devel. 2020:9:428-43.

21. Vernillet $L$, Greene $S A$, Kim HW, et al. Mass balance, metabolism, and excretion of cenobamate, a new antiepileptic drug, after a single oral administration in healthy male subjects. Eur J Drug Metab Pharmacokinet. 2020:45:513-22.

22. Bialer M, Johannessen SI, Levy RH, et al. Progress report on new antiepileptic drugs: a summary of the Eleventh Eilat Conference (EILAT XI). Epilepsy Res. 2013;103:2-30

23. Melnick S, Shin H, Roucard C, Glenn K. Cenobamate (YKP3089) dose-dependently reduced spike-and-wave discharges in the genetic absence epilepsy rats from strasbourg (GAERS) animal model. Presented at: Society for Neuroscience Annual Meeting, Chicago IL, October 19-23, 2019

24. Bialer M, Johannessen SI, Levy RH, et al. Progress report on new antiepileptic drugs: a summary of the Twelfth Eilat Conference (EILAT XII). Epilepsy Res. 2015;111:85-141.

25. Kasteleijn-Nolst Trenité D, Diventura BD, Pollard JR, et al. Suppression of the photoparoxysmal response in photosensitive epilepsy with cenobamate (YKP3089). Neurology. 2019;93:e559-67.

26. Chung SS, French JA, Kowalski J, et al. Randomized phase 2 study of adjunctive cenobamate in patients with uncontrolled focal seizures. Neurology. 2020;94:e2311-22.

27. Chung SS, French J, KrausS GL, et al. Long-term safety of adjunctive cenobamate in patients with uncontrolled focal seizures: open-label extension of a randomized clinical study. Neurology. 2020;94(Suppl. 15):1039.

28. Krauss GL, Klein P, Brandt C, et al. Safety and efficacy of adjunctive cenobamate (YKP3089) in patients with uncontrolled focal seizures: a multicentre, double-blind, randomised, placebo-controlled, dose-response trial. Lancet Neurol. 2020;19:38-48

29. Cramer JA, Mintzer S, Wheless J, Mattson RH. Adverse effects of antiepileptic drugs: a brief overview of important issues. Expert Rev Neurother. 2010;10:885-91.

30. Rosenfeld WE, Nisman A, Cho JW, Ferrari L. Efficacy of adjunctive cenobamate in patients with uncontrolled focal seizures based on number of concomitant antiepileptic drugs, seizure frequency, and epilepsy duration at baseline. Neurology. 2020;94(Suppl. 15):1025.
31. Klein P, Krauss GL, Aboumatar S, Kamin M. Long-term efficacy and safety of adjunctive cenobamate in patients with uncontrolled focal seizures: open-label extension of a randomized clinical study. Neurology. 2020;94(Suppl. 15):1008.

32. Ferrari L, Rosenfeld WE, Kamin M. Cenobamate adverse events by time of onset and dose from two randomized clinical studies in patients with uncontrolled focal seizures. Neurology. 2020;94(Suppl. 15):1019.

33. Sperling MR, Klein P, Aboumatar S, et al. Cenobamate (YKP3089) as adjunctive treatment for uncontrolled focal seizures in a large, phase 3, multicenter, open-label safety study. Epilepsia. 2020;61:1099-108.

34. Zaccara G, Franciotta D, Perucca E. Idiosyncratic adverse reactions to antiepileptic drugs. Epilepsia. 2007;48:1223-44.

35. Vernillet $L$, Kamin M. Drug-drug interactions between cenobamate and other antiepileptic drugs: results from phase I studies with carbamazepine, phenobarbital, phenytoin, and divalproex sodium. Presented at the Annual Meeting of the American Society for Clinic Pharmacology \& Therapeutics, Orlando FL, March 21-24, 2018.

36. Greene S, Kwak C, Kamin M, Vernillet L. The effect of cenobamate on the single dose pharmacokinetics of multiple cytochrome P450 probes using a cocktail approach in healthy subjects. Presented at the American Society for Clinical Pharmacology \& Therapeutics Annual Meeting, Washington DC, March 13-16, 2019

37. US Food and Drug Administration (FDA). Drug development and drug interactions: table of substrates, inhibitors and inducers. 2017. Available at: www.fda.gov/drugs/drug-interactions-labeling/ drug-development-and-drug-interactions-table-substratesinhibitors-and-inducers\#ttable2-2 (accessed August 14, 2020).

38. Gazzola DM, Balcer $\sqcup$, French JA. Seizure-free outcome in randomized add-on trials of the new antiepileptic drugs. Epilepsia. 2007:48:1303-7.

39. Halford JJ, Edwards JC. Seizure freedom as an outcome in epilepsy treatment clinical trials. Acta Neurol Scand. 2020;142:91-107.

40. Ben-Menachem E, Mameniskiene R, Quarato PP, et al. Efficacy and safety of brivaracetam for partial-onset seizures in 3 pooled clinical studies. Neurology. 2016;87:314-23.

41. Steinhoff BJ, Ben-Menachem E, Ryvlin P, et al. Efficacy and safety of adjunctive perampanel for the treatment of refractory partial seizures: a pooled analysis of three phase III studies. Epilepsia. 2013;54:1481-9.

42. Khan A, Fahl Mar K, Schilling J, Brown WA. Magnitude and pattern of placebo response in clinical trials of antiepileptic medications: data from the Food and Drug Administration 1996-2016. Contemp Clin Trials. 2018;64:95-100.

43. Goldenholz DM, Goldenholz SR. Response to placebo in clinical epilepsy trials-old ideas and new insights. Epilepsy Res.

2016;122:15-25. 